\title{
Les labyrinthes de l'esprit. Collections et bibliothèques à la Renaissance, sous la direction de R. Gorris Camos et A. Vanautgaerden (dir.)
}

\section{Maurizio Busca}

\section{(2) OpenEdition}

\section{Journals}

\section{Edizione digitale}

URL: http://journals.openedition.org/studifrancesi/10542

DOI: $10.4000 /$ studifrancesi. 10542

ISSN: 2421-5856

\section{Editore}

Rosenberg \& Sellier

\section{Edizione cartacea}

Data di pubblicazione: 1 décembre 2017

Paginazione: 538-539

ISSN: 0039-2944

\section{Notizia bibliografica digitale}

Maurizio Busca, «Les labyrinthes de l'esprit. Collections et bibliothèques à la Renaissance, sous la direction de R. Gorris Camos et A. Vanautgaerden (dir.)", Studi Francesi [Online], 183 (LXI | III) | 2017, online dal 01 février 2018, consultato il 27 janvier 2021. URL: http://journals.openedition.org/ studifrancesi/10542 ; DOI: https://doi.org/10.4000/studifrancesi.10542

Questo documento è stato generato automaticamente il 27 janvier 2021.

\section{cc) $($ ) $\ominus$}

Studi Francesi è distribuita con Licenza Creative Commons Attribuzione - Non commerciale - Non opere derivate 4.0 Internazionale. 


\title{
Les labyrinthes de l'esprit. Collections et bibliothèques à la Renaissance, sous la direction de R. Gorris Camos et A. Vanautgaerden (dir.)
}

\author{
Maurizio Busca
}

\section{NOTIZIA}

Les labyrinthes de l'esprit. Collections et bibliothèques à la Renaissance, sous la direction de Rosanna GORRIS CAMOS et Alexandre VANAUTGAERDEN, Genève, Droz, 2015, «Travaux d'Humanisme et Renaissance» 551, 673 pp.

1 Il presente volume riunisce gli atti di importanti convegni organizzati nel 2011 e nel 2012 a Montréal e a Cambridge dalla FISIER, dedicati alle Collections et bibliothèques de la Renaissance. I molteplici approcci proposti nei contributi raccolti, concernenti biblioteche pubbliche e private, permettono di esplorare diversi aspetti della «forma biblioteca» (che, come rileva VANAUTGAERDEN nel suo contributo liminare dal titolo $\mathrm{La}$ bibliothèque écartelée, pp. xv-Xxx, nel Rinascimento è intesa come biblioteca, collezione di libri e libro) e di seguire percorsi storici e archeologici che mirano a ricostruire biblioteche disperse o scomparse.

La prima delle sei sezioni in cui è suddiviso il volume introduce la tematica della creazione delle biblioteche. I due articoli che la compongono, di DELSAERDT e HENDRIX, trattano rispettivamente della fondazione della biblioteca pubblica di Anversa nel 1608 e delle biblioteche private di diversi umanisti italiani (Petrarca, Poggio Bracciolini, Marsilio Ficino, Pietro Bembo e Paolo Giovio) situate dall'A. lungo un percorso che conduce dal modello dello studio a quello del museo passando per quello dell'accademia. I contributi della seconda sezione, di LA CHARITÉ, CÉARD, MENINI e CAPPELLEN, partecipano al lavoro di ricostruzione della biblioteca rabelaisiana attraverso minuziose analisi che 
permettono di identificare le edizioni cui Rabelais ha attinto per la composizione delle sue opere (Ippocrate, Celio Rodigino, Plutarco, l'Antologia di Planude e il suo commento di Brodeau), un risultato raggiunto anche grazie allo studio degli apparati paratestuali di tali edizioni. La terza sezione guarda alla biblioteca di un altro autore capitale del Cinquecento francese, Montaigne. L'articolo di DEMONET illustra lo stato dell'arte del progetto di digitalizzazione Monloe, mentre quello di LEGROS tenta di definire un catalogo - parziale - della biblioteca di cui il bordolese disponeva in gioventù. SGATTONI si interessa ai rapporti fra Montaigne e censura, segnalando quali dei libri in suo possesso fossero stati messi all'Indice; PISTILLI, infine, si concentra in particolare su un volume posseduto dall'autore, il Ciceronianum Lexicon Grcecolatinum edito da Henri Estienne. La quarta sezione del volume è dedicata alle grandi biblioteche enciclopediche di umanisti francesi e italiani. RAUGEI, DE SMET, LIMPERHERZ e LINES illustrano le dinamiche di creazione, di trasformazione e di ampliamento delle collezioni di Pinelli, De Thou e Aldrovandi: veri centri di cultura, questi, che non si sono limitati a raccogliere libri ma che li hanno irradiati attraverso doni e scambi e, in alcuni casi, li hanno celati agli occhi dell'Inquisizione. Nella penultima sezione, ROUGET, BJAÏ e DEL SOLDATO propongono degli studi di tipo archeologico basati su cataloghi, lettere, inventari post mortem e altri documenti d'archivio sulle biblioteche di Philippe Desportes, Étienne Pasquier, François Rasse des Nœux, Simone Porzio e Benedetto Varchi. Tali lavori integrano i cataloghi provvisori esistenti con numerosi nuovi titoli (centinaia, nel caso di Desportes e Rasse des Nœux). La sezione conclusiva, a cura di GORRIS CAMOS, rievoca l'episodio dell'incendio della Biblioteca Nazionale di Torino del 1904 e, ripercorrendo le tappe dell'arduo lavoro di identificazione e restauro dei frammenti superstiti, illustra diversi casi di volumi andati perduti fra le fiamme o sopravvissuti - benché talvolta solo in maniera indiretta (attraverso trascrizioni), e spesso ridotti in condizioni pessime.

3 Completano il volume una bibliografia generale (pp. 549-596) e un indice dei nomi la cui estensione riflette la vastità dei contenuti (pp.597-667): strumenti, questi, che permettono allo studioso di orientarsi all'interno di questa preziosa raccolta di studi di cui forniamo qui l'indice.

4 Partie I («Naissance des bibliothèques»): Pierre DELSAERDT, À l'ombre de l'Ambrosienne: les débuts de la bibliothèque publique d'Anvers en 1608 et 1609, pp. 3-23; Harald HENDRIX, Italian humanists at home: villas, libraries, and collections, pp. 25-42; Partie II («La bibliothèque retrouvée de Rabelais»): claude LA CHARITÉ, La bibliothèque hippocratique de Rabelais dans "Gargantua", l'“Almanach" de 1535, le "Tiers livre" et le "Cinquiesme livre", pp. 45-74; Jean CÉARD, Rabelais antiquaire: les "Lectiones antiqua" de Coelius Rhodiginus et le "Tiers livre", pp. 75-87; Romain MENINI, Le dernier Plutarque de Rabelais, pp. 89-104; Raphaël CAPPELLEN, Rabelais lecteur des "Epigrammatum Groecorum libri VII" commentés par Jean Brodeau (1549), pp.105-127; Partie III («La bibliothèque de Montaigne»): Marie-Luce DEMONET, Éphémérides de la bibliothèque de Montaigne, pp.131-153; Alain LEGROS, À la recherche des premiers livres de Montaigne, pp. 155-172; Marco SGATTONI, Les "libri prohibiti" de Montaigne, pp.173-192; Barbara PISTILLI, Dai «grecs» agli essais: un lessico greco-latino ignorato della «librairie» di Montaigne, pp. 193-209; Partie IV («Bibliothèques encyclopédiques»): Anna Maria RAUGEI, Gian Vincenzo Pinelli (1535-1601) ses livres, ses amis, pp. 213-227; Ingrid A. R. DE SMET, Des livres pour de Thou (et Pinelli): collectionneurs, livres clandestins et sillons confessionnels, pp. 229-253; Karen LIMPER-HERZ, Bindings from the library of Jacques Auguste de Thou in the Bibliotheca Grenvilliana, pp. 255-302; David A. LINES, A library for teaching and 
study: Ulisse Aldrovandi's aristotelian texts, pp.303-379; Partie V («Bibliothèques recomposées»): François ROUGET, Éléments nouveaux pour la reconstruction de la bibliothèque de Philippe Desportes, pp. 383-401; Denis BJAÏ, La bibliothèque d'Étienne Pasquier au miroir de sa correspondance, pp. 403-419; François ROUGET, Le chirurgien et ses livres: complément d'enquête sur la bibliothèque de François Rasse des Neux (ca 1525-1587), pp. 421-471; EVa DEL SOLDATO, "In pulvere, quasi neglecta": the libraries of Simone Porzio and Benedetto Varchi, pp. 473-485; Partie VI («Renaissance d'une bibliothèque»): Rosanna GORRIS CAMOS, “Una notte d'inferno": autour de l'incendie de la Bibliothèque nationale de Turin, livres détruits, livres rescapés, pp. 489-547. 